\title{
FARMA: Uma Ferramenta de Autoria para Objetos de Aprendizagem de Conceitos Matemáticos
}

\author{
Diego Marczal ${ }^{1,2}$, Alexandre Direne (orientador) ${ }^{1}$, \\ Andrey Ricardo Pimentel (coorientador) ${ }^{1}$, Eleandro Maschio ${ }^{2}$ \\ ${ }^{1}$ Programa de Pós-Graduação em Informática \\ Universidade Federal do Paraná (UPFR) \\ Curitiba - PR - Brasil \\ ${ }^{2}$ Coordenação do Curso de Tecnologia em Sistemas para Internet \\ Universidade Tecnológica Federal do Paraná (UTFPR) \\ Guarapuava - PR - Brasil \\ marczal, eleandrom@utfpr.edu.br, alexd, andrey@inf.ufpr.br
}

\begin{abstract}
The problem of building learning objects with authoring tools is exposed in a critical way. The main limitations of current approaches to mathematics teaching with learning objects are pointed out in the literature review. A web authoring tool, focusing on the concept of techno-educational mobility, is presented as an alternative to existing research. The mistakes of the learner are highlighted as a central cognitive approach to the design and implementation of the FARMA prototype tool. Experimental results suggest that the framework of method and tool achieves learning benefits.
\end{abstract}

Resumo. O problema de construção de objetos de aprendizagem com ferramentas de autoria é exposto de maneira crítica. As principais limitações das abordagens atuais do ensino de matemática com objetos de aprendizagem são apontadas na resenha literária. Uma ferramenta web de autoria, com enfoque no conceito de mobilidade tecnológico-educacional, é apresentada como alternativa às pesquisas existentes. O erro do aprendiz é destacado como abordagem cognitivista no projeto e implementação do protótipo FARMA. Resultados experimentais mostram que o arcabouço de método e ferramenta atinge benefícios educacionais.

\section{Introdução}

A avaliação da educação no Brasil é realizada constantemente pelo Instituto Nacional de Estudos e Pesquisas Educacionais "Anísio Teixeira" (INEP). Dentre as avaliações realizadas, a Avaliação Nacional da Educação Básica (Aneb) é a única que abrange o Ensino Fundamental e Médio, sendo aplicada em áreas da Língua Portuguesa, com foco em interpretação de texto, e Matemática, centrada na resolução de problemas.

Da avaliação realizada em 2011 para área da Matemática, em âmbito nacional, o nível alcançado pelos anos iniciais do Ensino Fundamental foi 4 e pelos anos finais foi 6 , de 13 níveis (0 à 12). Já para o Ensino Médio, o resultado atingido pelo $3^{\mathrm{o}}$ (terceiro) ano/série foi 0 de 5 níveis (0 à 4). Na última avaliação, realizada em 2013, em âmbito nacional, os mesmos níveis se mantiveram. 


\section{CBIE-LACLO 2015}

Anais dos Workshops do IV Congresso Brasileiro de Informática na Educação (CBIE 2015)

Esses resultados indicam que existe uma grande deficiência no ensino e aprendizagem da Matemática, pois os aprendizes sequer alcançaram $50 \%$ dos níveis avaliados. Com isso, destaca-se a importância da busca por novas abordagens de ensino e de aprendizagem da Matemática, além do aperfeiçoamento das já existentes. Outras pesquisas, como a de [Flores 2011], também destacam dificuldades no ensino e aprendizado de Matemática por meio de resultados de avaliações nacionais.

Segundo estudos de [Notare and Behar 2010], os professores conhecem as dificuldades dos aprendizes no ensino da Matemática e constantemente buscam novas alternativas pedagógicas. Porém, ainda existem grandes objeções em alterar as metologias tradicionais de ensino. Isso evidencia a necessidade do desenvolvimento de novas abordagens metodológicas para o ensino e aprendizagem da Matemática. Nesse sentido, uma das abordagens de maior destaque é o uso das tecnologias digitais no processo de ensino-aprendizagem. No que diz respeito à Matemática, essas tecnologias ajudam o aprendiz principalmente por meio da simulação computacional, em que situações do mundo real permitem a visualização de conceitos por meio de uma representação intuitiva.

Porém, a criação de softwares educacionais (e.g. Sistemas Tutores Inteligentes (STIs) e Objetos de Aprendizagem (OAs)) que promovam intuitividade nas simulações não é uma tarefa simples. [Murray et al. 2003] observou 20 ferramentas de autoria destacando que elas são complexas, sendo difícil imaginar seu uso, por professores, sem um suporte contínuo. Ainda, segundo Murray, a produção de um STIs que ocupe o tempo de 1 hora em sala de aula, exige um esforço de 300 a 1.000 horas de trabalho de autoria.Pesquisas tentaram minimizar esse esforço, sendo que as mais bem sucedidas foram aquelas que propuseram ferramentas de autoria como mecanismos centrais de seus resultados científicos. No entanto, tais ferramentas ainda são de difícil acesso e compreensão por parte de muitos autores (professores) de conteúdos [Flores 2011].

Em paralelo, a web vem se tornando um dos meios mais importantes para a disponibilização e execução de OAs, além de oferecer recursos para individualizar o perfil de aprendizes e monitorá-los por longos períodos de tempo. Com esse crescimento, fica evidente a necessidade de ferramentas de autoria totalmente voltadas para a web, pois com elas o professor pode construir softwares educacionais e disponibilizá-los ao aprendiz de forma a promover até mesmo o seu uso sob aspectos de mobilidade tecnológico-educacional [Direne et al. 2012].

Em contrapartida, durante os estudos, o aprendiz pode errar por descuido ou pela falta de conhecimento de conceitos. No segundo caso, a remediação do erro pode ser demorada e até depender da ajuda do professor. Consequentemente, uma nova tentativa de resolver o mesmo exercício poderá exigir algum tempo (talvez várias semanas), pois dependerá de uma reestruturação cognitiva do aprendiz. Por isso, o erro no processo de aprendizagem não pode ser negligenciado, mesmo em OAs construídos por professores com pouco conhecimento em programação.

Assim, o objetivo deste trabalho foi a pesquisa, desenvolvimento e aplicação de uma ferramenta de autoria web com foco na construção de OAs destinados ao ensino de conceitos matemáticos. Buscou-se possibilitar que o erro cometido pelo 


\section{CBIE-LACLO 2015}

Anais dos Workshops do IV Congresso Brasileiro de Informática na Educação (CBIE 2015)

aprendiz fosse utilizado como uma oportunidade de aprendizagem, isso por meio de mecanismos que permitissem remediação e retroação a erros a curto e longo prazo. Adicionalmente, que possa ser utilizada por professores com conhecimentos básicos em informática.

Para avaliar a ferramenta proposta, e principalmente seu mecanismo de retroação a erros (diferencial do trabalho), foram realizados experimentos com alunos e professores de escolas públicas. Os resultados indicaram que a ferramenta teve um boa aceitação por parte dos professores e que os alunos que usaram OAs construídos com ela e fizeram uso do mecanismo de retroação a erros apresentaram um aumento significativo na aquisição de conhecimento quando comparados com os que não usaram o mecanismo de retroação.

\section{Resenha Literária}

Os Sistemas Tutores Inteligentes (STIs) são ferramentas educativas que têm por objetivo assistir aprendizes e professores no processo de aquisição e transmissão de conhecimento. Uma das características de destaque dos STIs é a possibilidade de trabalhar com os erros dos aprendizes. A partir desses erros, os STIs podem identificar as dificuldades individuais do aprendiz e então fornecer um feedback apropriado para corrigir desvios na formação de conceitos [Murray et al. 2003]. Tais ações são baseadas em teorias de aprendizagem, como aquelas propostas pelo ACT (Adaptive Control of Thought) que se fundamentam em manter o aprendiz em uma linha de aprendizagem ideal, mesmo que restrita [Ritter et al. 2007].

O Geometry Tutor é um exemplo de STI para ensino da geometria de [Anderson et al. 1985] que explorou os erros dos aprendizes por meio de um módulo de diagnóstico automático de erros. O conhecimento do sistema tutor é baseado em regras de produção, sendo que não possui modelagem dinâmica do aprendiz, porém utiliza um modelo ideal que representa todo o conhecimento que supostamente o aprendiz deve adquirir.

Outro STI é o PAT2Math [Seffrin et al. 2012], voltado para o ensino de álgebra elementar, com equações de $1^{\circ}$ e $2^{\circ}$ graus de uma incógnita. Seu principal componente é o PATequation, um módulo de resolução de equações que permite ao aluno resolver uma equação passo a passo. O módulo provê ao aluno a correção e feedback em tempo real de cada passo fornecido. Apesar de ter apresentado sucesso em suas pesquisas, o sistema não guarda nem monitora os erros para mostrá-los aos aprendizes e tutores a longo prazo.

As teorias da ACT, apesar de bem sucedidas em STIs, limitam-se a condições em que o feedback é explícito, imediato e em que os erros do aprendiz possam ser inteiramente identificados. Além disso, existem poucas pesquisas sobre seu uso em micromundos e objetos de aprendizagem, principalmente sobre ferramentas de autoria para gerar material capaz de registrar e monitorar tais abordagens dos erros.

\subsection{Ambientes de Autoria para STIs}

Autoria de material digital, ou simplesmente autoria, em Educação é o processo de organizar ou produzir uma sequência de informações de modo a compor um material para o ensino-aprendizagem, (e.g., um Sistemas Tutores Inteligentes, Objeto de 


\section{CBIE-LACLO 2015}

Anais dos Workshops do IV Congresso Brasileiro de Informática na Educação (CBIE 2015)

Aprendizagem) que possibilite a interação do usuário com partes de um domínio específico. Com isso, pode-se definir uma ferramenta de autoria como um software em que um tutor humano possa manipular, criar, alterar ou excluir sessões de ensino.

No passado, pesquisas foram realizadas nesta área para o desenvolvimento de ferramentas de autoria para STIs [Murray et al. 2003]. Um exemplo recente de ferramenta de autoria, o EDUCA, permite a construção de conteúdos educacionais personalizados que podem ser utilizados de maneira colaborativa. Em seu trabalho, [Cabada et al. 2011] propõe uma abordagem para determinar o estilo de aprendizado do indivíduo usando rede de mapas auto-organizáveis (Kohonen neural networks). A rede é atualizada por questionários fornecidos ao aprendiz, durante a interação com o conteúdo.

O CTAT (Cognitive Tutor Authoring Tools) [Aleven and Sewall 2010] é um projeto com o objetivo de prover um conjunto de ferramentas de autoria para o desenvolvimento de dois tipos principais de STIs: cognitivos e baseados em exemplos. O primeiro exige autores com conhecimento em programação e inteligência artificial. Já o segundo, com os mesmos recursos do primeiro, não requer nenhum conhecimento em programação de computadores.

Já o IGeom de [Isotani and Brandão 2004] é um software para Geometria Dinâmica (GD) que permite realizar todas as operações básicas da Geometria. Um dos módulos oferecidos por essa ferramenta permite a autoria de exercícios envolvendo a GD, assim como a correção automática pelo sistema. O uso do CTAT permite que, durante a resolução de um problema, o tutor possa identificar os passos do aprendiz e lançar algum tipo de feedback ou sugestão. Apesar do sistema oferecer feedback imediato sobre os erros dos aprendizes, não destaca a exploração e retroação ao contexto erro, nem pelo professor nem pelo aprendiz.

\subsection{Ambientes de Autoria para Objetos de Aprendizagem}

Os OAs também podem ser vistos como um recurso cognitivo para auxiliar e ampliar o ensino e aprendizagem com características peculiares, dentre as quais destacam-se a reusabilidade, granularidade, acessibilidade e interoperabilidade. No quesito ferramentas de autoria para Objetos de Aprendizagem, pode-se destacar algumas ferramentas como o CourseLab, eXe Learning, HotPotatoes, Microsoft LCDS e MyUdutu, as quais foram destacadas e avaliadas em pesquisas anteriores apresentadas por [Battistella and von Wangenheim 2011].

Todas essas ferramentas são bem sucedidas para a produção de arquivos digitais em diferentes mídias, tais como texto, imagem, som e vídeo. Entretanto, os objetos criados a partir dessas ferramentas fornecem pouca interação com o aprendiz, principalmente no que se refere ao sentido crítico da carência de recursos de avaliação formativa, atuando apenas na simples avaliação somativa. Em particular, os ambientes citados não tratam explicitamente os erros cometidos pelo aprendiz como uma importante fonte de apoio ao aprendizado autônomo. Uma tentativa de trabalhar com erro em ferramentas de autoria para Objetos de Aprendizagem foi apresentada por [Stamey and Saunders 2005], mas não promove condições de exploração dos erros dos aprendizes, apenas opções de reestruturação da questão. 


\section{CBIE-LACLO 2015}

Anais dos Workshops do IV Congresso Brasileiro de Informática na Educação (CBIE 2015)

\section{Fundamentos da Solução}

Uma das características mais importantes na construção de sistemas para o amparo do ensino está no desenvolvimento do conteúdo, pois a dificuldade na sua elaboração pode ser um fator determinante na utilização ou não de uma ferramenta. Por isso, um ambiente de autoria, além de permitir criar conteúdos de qualidade, interativos e que produzam uma avaliação formativa, devem ser fáceis de utilizar.

Por outro lado, em uma sessão de estudo, o aprendiz está sujeito a erros, seja por descuido ou por falta de conhecimento de conceitos. Assim, é importante se beneficiar deste fato para promover a construção do conhecimento do aprendiz. Por meio do erro, pode-se oferecer duas principais formas de aprendizado: a remediação de erros, na qual o aprendiz recebe um feedback imediato sobre a falha, permitindo a sua recuperação e a continuação dos estudos; e a retroação aos erros, em que o aprendiz pode explorar os erros e, a partir disso, tentar refazer a interação onde o erro foi cometido para entender seu motivo e então solucioná-lo.

Tendo isso em vista, foi proposta uma ferramenta de autoria denominada FARMA (Ferramenta de Autoria para a Remediação de erros com Mobilidade na Aprendizagem). Ela permite a construção de exercícios voltados ao aprendizado de conceitos de indução analítica que envolvem expressões aritméticas e/ou algébricas. Suas principais características são a de constituir uma ferramenta simples e objetiva para o desenvolvimento de OAs altamente interativos e promover uma aprendizagem por erros, dos quais são registrados desde a hora em que ocorrem até seus respectivos contextos. Assim, os aprendizes podem, posteriormente, explorar seus próprios erros, além de permitir que os professores tenham acesso integrado ou individual aos aprendizes de uma turma virtual. A dinâmica de funcionamento proposta para esta ferramenta pode ser observada na Figura 1.

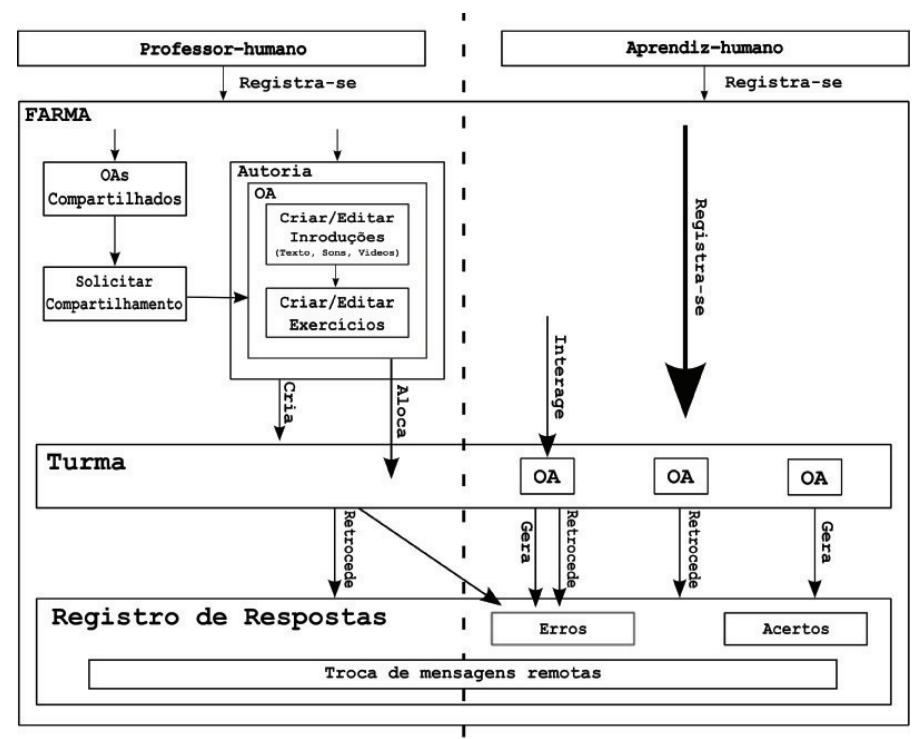

Figura 1. Dinâmica de funcionamento do arcabouço

Conforme pode ser visualizado na Figura 1, o processo inicia a partir de dois atores principais, o professor e o aprendiz, os quais primeiramente devem se registrar 


\section{CBIE-LACLO 2015}

Anais dos Workshops do IV Congresso Brasileiro de Informática na Educação (CBIE 2015)

na ferramenta para posterior interação. Na perspectiva do professor, ele pode começar observando os OAs já desenvolvidos por outros professores e, quando se interessar por algum deles, pode solicitar o compartilhamento e então usá-lo da maneira como o visualizou ou editar conforme necessário. Outro possível caminho que o professor pode seguir é criar seu próprio OA. Um OA, da maneira como foi projetado, será composto por introduções a conteúdos seguidas de exercícios interativos. Para aplicação dos OAs desenvolvidos junto aos aprendizes, o professor primeiramente deve criar uma turma virtual e alocar os objetos para que os aprendizes interajam.

Para os aprendizes interagirem com os OAs, antes devem se matricular em uma turma, geralmente indicada por um professor. Na turma matriculada, estarão todos os OAs vinculados pelo professor. Durante a interação do aprendiz, a ferramenta registra o histórico do aprendiz com o OA, incluindo seções de estudo em que houve envio de respostas corretas e incorretas. Posteriormente, tanto o professor quanto o aprendiz podem retroceder aos erros cometidos e podem também tentar refazer os exercícios a partir do seu erro. Durante esse processo, também é possível a discussão entre professores e aprendizes sobre suas respostas, principalmente sobre seus erros.

\subsection{Remediação por Retroação}

Durante seus estudos, o aprendiz pode errar por descuido ou pela falta de conhecimento de conceito. No segundo caso, a remediação do erro pode ser demorada e até depender da ajuda do professor. Consequentemente, uma nova tentativa de resolver o mesmo exercício poderá exigir algum tempo (talvez várias semanas) pois dependerá de uma reestruturação cognitiva do aprendiz. No entanto, com a possibilidade de o aprendiz retroagir ao contexto do erro de maneira autônoma (que só depende da interação com a FARMA), é possível alcançar uma dinâmica de autoestudo fundamentalmente distinta das existentes nos OAs construídos até hoje.

Tal comportamento do aprendiz só é possível se implementado um mecanismo de modelagem de longo prazo do aprendiz, acoplado à base de exercícios criados pelo autor do material eletrônico. Com isso, o erro deixa de ser apenas uma forma de avaliar somativamente o usuário para se tornar a modalidade concreta de avaliação formativa na construção do conhecimento, tal como advogou a psicologia cognitivista de Piaget, Papert e seus seguidores. Em outras palavras, a alternância entre a realização de novos exercícios e a restauração do contexto de antigos enunciados que foram resolvidos com erro, parece se alinhar com as visões de futuro que estão nas mais promissoras perspectivas de mudança pedagógica qualitativamente relevante.

\section{Arquitetura Funcionalista}

O objetivo desse seção é apresentar a arquitetura funcionalista da FARMA, que foi desenvolvida para consolidar os fundamentos de aprendizagem descritos na Seção 3. Conforme apresentado na Figura 4, a arquitetura possui três estágios principais: autoria, interação e acompanhamento.

Módulo de Autoria: Fornece todo arcabouço necessário para a construção de objetos de aprendizagem matemáticos. Ele permite a elaboração da parte teórica de um OA, chamada de introduções, e também da parte prática, que são os exercícios. 


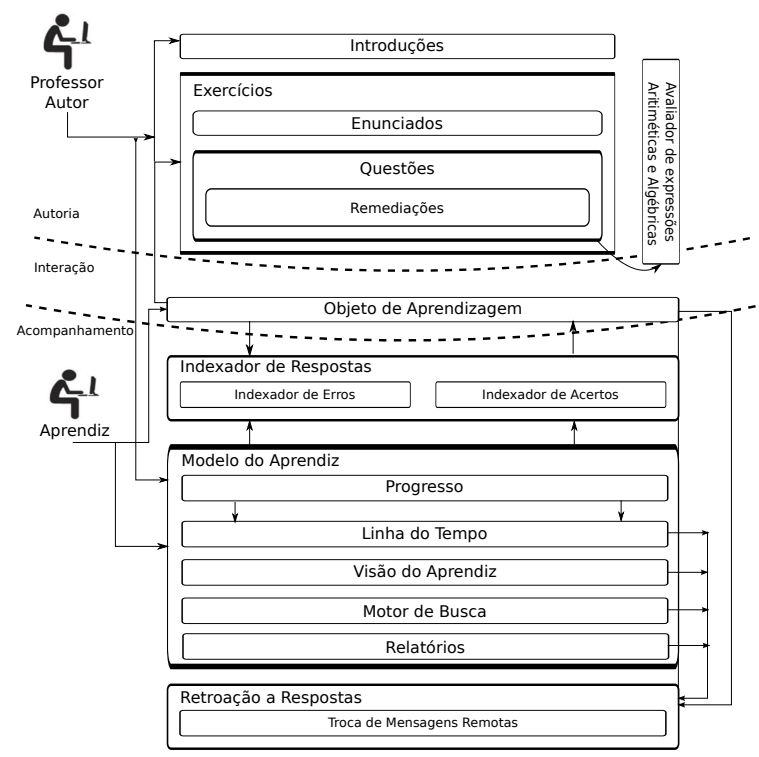

Figura 2. Arquitetura funcionalista da FARMA

Módulo de Interação: Interface entre o aprendiz e o OA criado pelo professorautor. Este módulo realiza a junção das introduções, exercícios, questões e remediações a curto prazo, definidas pelo professor-autor, para compor um OA.

Módulo de Acompanhamento: Por meio desse módulo, o professor-autor pode realizar uma avaliação formativa de um aprendiz. Para isso, o módulo subdivide-se em 3 partes principais, são elas: (a) Indexador de respostas, que salva todas as respostas enviadas pelo aprendiz, sejam elas erradas ou corretas. (b) Modelo do aprendiz, que apresenta algumas informações ao professor-autor sobre a interação do aprendiz, como: (1) o progresso do aprendiz; (2) a linha do tempo do aprendiz; (3) a visão do aprendiz sobre o OA e (d) relatórios sobre interação; (c) Retroação a respostas, que permite o professor-autor retroceder ao contexto exato de uma resposta do aprendiz, analisar e promover discussões com o aprendiz sobre a mesma.

\section{Validação da FARMA e do seu Mecanismo de Retroação}

O primeiro uso real da FARMA foi em um experimento que resultou da parceria com a pesquisa de [Leite et al. 2013]. Neste, foi avaliado o módulo de autoria por meio da construção de 4 OAs, e também o mecanismo de remediação de erros a curto prazo. O diferencial do experimento foi o uso da s Múltiplas Representações Externas (MREs) para realização das remediações a erros de curto prazo.

O experimento contou com participação de 60 alunos. Os resultados indicaram que os alunos que utilizaram o OA com remediação a erros por meio de MREs tiveram ganhos significativos na aquisição de conceitos, quando comparado com os que usaram o OA sem remediação a erros por meio de MREs. Detalhes de ambas as aplicações estão em [Leite et al. 2013]

Como segundo experimento, foi realizada uma oficina com 10 alunos do PIBIC da Matemática da Universidade Federal do Paraná (UFPR). Eles, além de conhecerem a ferramenta, poderiam também divulgá-la a outros professores, pois dentre as atividades do PIBIC estão o acompanhamento e reforço de conteúdos matemáticos com alunos de escolas público. Ao final da oficina, foi aplicado um 


\section{CBIE-LACLO 2015}

Anais dos Workshops do IV Congresso Brasileiro de Informática na Educação (CBIE 2015)

questionário, onde os participantes mostraram interesse em utilizar a FARMA e também seu mecanismos de retroação.

Um terceiro experimento foi realizado com intuito de avaliar formalmente o mecanismo de retroação a erros como recurso adicional para aquisição de conhecimento do aprendiz. Para isso, foi elaborado um experimento e consequente análise dos dados por meio do método estatístico não paramétrico Wilcoxon-Mann-Whitney.

O experimento foi realizado em 3 turmas do ensino médio do Centro Estadual de Educação Profissional de Curitiba, contando inicialmente com um total de 70 alunos divididos em dois grupos (Grupo Controle (GC) e Grupo Experimental (GE)) a partir do resultado de um pré-teste. De maneira geral, o experimento foi composto por um pré-teste, a interação com um OA (GE interagiu com OA tendo o mecanismo de retroação a erros habilitado e GC sem o mecanismo de retroação a erros) e, por fim, a aplicação de um pós-teste.

No gráfico da Figura 3, tem-se a comparação entre as amostras Controle e Experimental. Destaca-se que as diferenças entre os pré-testes dos dois Grupos não foram significantes (GE com média de 0,6750 e o GC com média de 0,6333, com $\mathrm{W}=$ $64,5$ e $p$-valor atribuído $=0,6809)$, indicando que os grupos possuem mesmo nível de conhecimento. Na verificação entre os pós-testes, houve uma diferença significativa, que também pode ser observada no Gráfico. Isso comprova que as notas do pós-teste do GE (média de 0,6917) foram melhores que as notas do pós-teste do GC (média de 0,5567$)$ com $\mathrm{W}=37,5$, p-valor atribuído $=0,0483$.
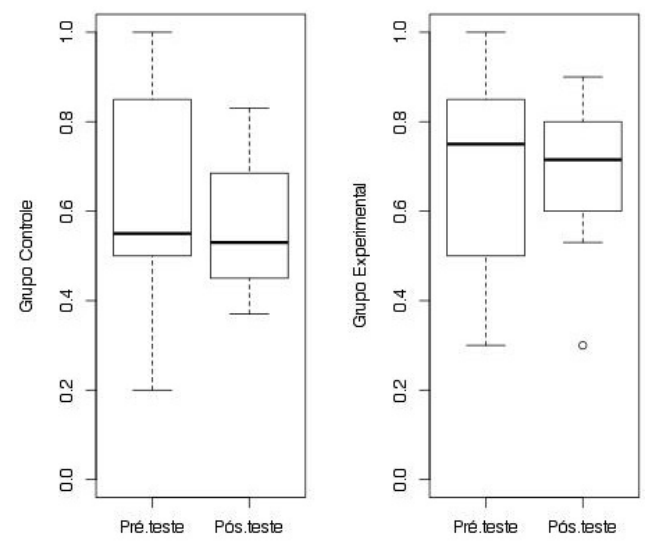

Figura 3. Comparação entre as amostras Controle e Experimental

\section{Resultados}

Em alinhamento aos objetivos do trabalho, apresenta-se nesta seção os principais resultados alcançados pela pesquisa, a saber: (1) Elaboração e implementação de uma abordagem para trabalhar com os erros dos aprendizes fundamentalmente diferente daquelas encontradas nas pesquisas da área, que é a retroação a erros; (2) A ferramenta de autoria web FARMA, para a criação de OA matemáticos; (3) Uso da remediação a curto e longo prazo em uma mesma ferramenta de autoria; (4) Apresentação dos dados gerados pela interação do aprendiz com o ambiente, de forma a compor o modelo do aprendiz; e, (5) Por fim, a autoria junto ao mecanismo de retroação a erro, neste não se tem apenas um OA a ser explorado, mas sim um 


\section{CBIE-LACLO 2015}

Anais dos Workshops do IV Congresso Brasileiro de Informática na Educação (CBIE 2015)

grande número de possibilidades de OAs que podem ser criados e compartilhados, a partir da ferramenta, e sem nenhum esforço adicional o professor e o aprendiz podem usufruir do mecanismo de retroação a erros.

Elencam-se também os resultados de pesquisas relacionadas e/ou derivadas do presente trabalho, são elas: (1) Classificação Automática de Erros de Aprendizes Humanos no Processo de Indução Analítica, este trabalho foi uma pesquisa de mestrado desenvolvida por [Gustavo Bazzo 2011]; (2) Arquitetura para Remediação de Erros Baseada em Múltiplas Representações Externas, trata-se de uma pesquisa de doutorado desenvolvida por [Leite et al. 2013]. O objetivo principal foi definir uma arquitetura com o propósito de explorar uma abordagem para o uso das múltiplas representações externas (MREs), no processo de remediações de erros matemáticos durante o uso de Objetos de Aprendizagem; (3) Pesquisa de doutorado, com o propósito de adaptar a FARMA para o ensino de programação de computadores, onde a ferramenta disponibiliza a manipulação visual e semântica e também permite a organização e a classificação dos erros de maneira semi-automática, realizada em um momento pela ferramenta e em outro pelo professor [Kutzke and Direne 2014]; (4) Sequenciamento de Exercícios Guiado por Rating em Sistemas Tutores Inteligentes, pesquisa de doutorado que utiliza a FARMA para elaboração de OAs e aplicação das técnicas de sequenciamento de exercícios; (5) Elaboração e Aplicação de OAs para Avaliação da FARMA, trabalho de conclusão de curso que visa o desenvolvimento de vários OAs em parceira com professores do ensino básico e médio do Centro Estadual de Educação Profissional de Curitiba, Paraná.

\section{Considerações Finais}

Acredita-se que esta pesquisa abre espaço para que sejam retomados os estudos sobre uso do erro como fonte de oportunidade para o ensino e aprendizagem, principalmente em uma nova perspectiva proposta por essa pesquisa, a de retroação a erros. Os impactos deste recurso são mostrados pelos resultados estatístcos positivos dos experimentos de avaliação realizados.

Há ainda um total de cinco trabalhos derivados: três de doutorado (um terminado e dois em andamento); um de mestrado (em andamento) e um TCC concluído. Destaque-se também que a FARMA saiu do laboratório de pesquisa e está sendo usada em ambientes reais de ensino, constituindo assim uma contribuição relevante para a área de Informática Aplicada a Educação. Os trabalhos futuros apontam para uma pesquisa na mineração dos erros dos aprendizes, assim como na forma de apresentação para professores e aprendizes.

\section{Referências}

Aleven, V. and Sewall, J. (2010). Hands-on introduction to creating intelligent tutoring systems without programming using the cognitive tutor authoring tools (CTAT). In Proceedings of the 9th International Conference of the Learning Sciences-Volume 2, pages 511-512. International Society of the Learning Sciences.

Anderson, J. R., Boyle, C. F., and Yost, G. (1985). The geometry tutor. In IJCAI, pages $1-7$. 


\section{CBIE-LACLO 2015}

Anais dos Workshops do IV Congresso Brasileiro de Informática na Educação (CBIE 2015)

Battistella, P. E. and von Wangenheim, A. (2011). Avaliação de ferramentas de autoria gratuitas para produção de Objetos de Aprendizagem no padrão SCORM. Revista Brasileira de Informática na Educação, 19(3):16-28.

Cabada, R. Z., Estrada, M. L. B., and García, C. A. R. (2011). Educa: A web 2.0 authoring tool for developing adaptive and intelligent tutoring systems using a Kohonen network. Expert Systems with Applications, 38(8):9522 - 9529.

Direne, A., Silva, W., Silva, F., Peres, L., Kutzke, A., Marczal, D., Barros, G., Moura, L., and Bazzo, G. (2012). Aprofundamento da mobilidade tecnológicoeducacional por meio de jogos intelectivos. In Anais do DesafIE-2012, pages 1-10. SBC.

Flores, M. L. P. (2011). Metodologia para criar Objetos de Aprendizagem em matemática usando a combinação de ferramentas de autoria. Tese de doutorado, Universidade Federal do Rio Grande do Sul - UFRS.

Gustavo Bazzo, Alexandre Direne, D. M. (2011). Classificação automática de erros de aprendizes humanos do processo de indução analítica. In Anais do SBIE-2011. SBIE.

Isotani, S. and Brandão, L. d. O. (2004). Ferramenta de avaliação automática no iGeom. In Anais do Simpósio Brasileiro de Informática na Educação, volume 1, pages 319-328.

Kutzke, A. R. and Direne, A. (2014). Mediação do erro na educação: um arcabouço de sistema para a instrumentalização de professores e alunos. In Anais do Simpósio Brasileiro de Informática na Educação, volume 25, pages 737-746.

Leite, M. D., Marczal, D., and Pimentel, A. R. (2013). Objeto de Aprendizagem Pitágoras: uma aplicação do uso de múltiplas de representações externas na remediação de erros matemáticos. In DesafIE! - II Workshop de Desafios da Computação Aplicada à Educação do XXXIII Congresso da Sociedade Brasileira de Computação, pages 1434-1442.

Murray, T., Blessing, S., and Ainsworth, S. (2003). Authoring tools for advanced technology learning environments: Toward cost-effective adaptive, interactive and intelligent educational software. Springer.

Notare, M. R. and Behar, P. A. (2010). Comunicação e aprendizagem matemática on-line: Um estudo com o editor científico rooda exata. Revista Brasileira de Informática na Educação, 18(1).

Ritter, S., Anderson, J. R., Koedinger, K. R., and Corbett, A. (2007). Cognitive tutor: Applied research in mathematics education. Psychonomic bulletin $\&$ review, $14(2): 249-255$.

Seffrin, H., Rubi, G., Ghilardi, C., Morais, F., Jaques, P., Isotani, S., and Bittencourt, I. I. (2012). Dicas inteligentes no sistema tutor inteligente PAT2Math. In Anais do Simpósio Brasileiro de Informática na Educação, volume 23.

Stamey, J.W., J. and Saunders, B. (2005). Designing intelligent learning objects. In Advanced Learning Technologies, 2005. ICALT 2005. Fifth IEEE International Conference on, pages 323-325. 